\title{
EL PROBLEMA DEL NINO PREMATURO EN LA ZONA NORTE DE SANTIAGO
}

\author{
Dr. HUMBERTO GARCES \\ Cátedra de Pediatria del Prof. Arturo Scroggie. \\ Hospital "Roberto del Rio". Santiago.
}

El hecho de que en Chile no se haya dado la debida importancia, sino en estos últimos años, al problema de la prematurez, que preocupa a los pediatras de casi todos los países del mundo, tiene su explicación. Nuestras altas tasas de mortalidad infantil nos obligaba a concentrar la lucha hacia aquellos rubros que eran sus causas más importantes: los trastornos nutritivos y las infecciones, especialmente las de localización digestiva $\mathrm{y}$ respiratoria. Los esfuerzos de la pediatría nacional se habían concentrado hasta el momento, muy justificadamente por cierto, en prevenir los primeros y en curar las últimas.

A medida que la tasa de mortalidad infantil ha ido descendiendo, se ha podido apreciar la importancia cada vez mayor que tiene la mortalidad neonatal $y$, dentro de ella, su causa principal: la prematurez. En paises con alto standard de vida y baja morțalidad infantil, como Sue- cia por ejemplo, se ha visto que la mortalidad infantil casi coincide con la mortalidad neonatal y que ésta es producida en la mitad de los casos por la prematurez. Al respecto, bástenos citar a Wallgren ${ }^{4}$, quien en un reciente trabajo dice textualmente: "La mortalidad infantil en Suecia es la más baja del mundo, habiendo alcanzado una tasa de $2 \%$ en 1951 . Este $2 \%$ es, poco más o menos, idéntico a la mortalidad neonatal. La causa más frecuente de la mortalidad neonatal en la prematurez, que constituye el $\mathbf{5 0 \%}$ de todas sus causas. Para mejorar ésta, es, pues, sobre todo preciso prevenir los nacimientos precoces y mejorar las condiciones de vida de los niños nacidos prematuros".

\section{REFERENCIAS DEMOGRÁFICAS}

La circunstancia de haber tenido a nuestro cargo el Servicio de Prematuros del Hospital de Niños "Roberto del Río" 
durante los 3 últimos años, nos ha permitido palpar de cerca la gravedad del problema de la prematurez en nuestro medio, llevándonos a realizar una investigación estadística que nos permitiera medir su intensidad en la Zona Norte de Santiago y, sobre esta base, proceder a elaborar un programa de atención del niño prematuro, coordinando los medios asistenciales existentes en ella.

Entendemos por Zona Norte de Santiago, la parte del departamento del mismo nombre que queda situada al norte del río Mapocho. Sus límites son: la cuesta de Chacabuco por el norte, el cerro San Cristóbal y adyacentes por el este, el límite poniente de las Comunas de Renca y Quilicura por el oeste y el río Mapocho por el sur. Comprende las circunscripciones de Independencia y Recoleta de la comuna de Santiago y las comunas de Renca, Quilicura, Conchalí, Colina, Lampa y Til-Til. Incluye, por lo tanto, sectores urbanos, suburbanos y rurales del departamento de Santiago, estos últimos con fáciles medios de acceso a los centros asistenciales. Su población alcanza a 300 mil habitantes, en su mayoria perteneciente a la clase obrera.

El Hospital de Niños "Roberto del Rio" es el principal centro de asistencia infantil de la Zona. Desde su traslado al nuevo edificio, en 1939, por iniciativa del Dr. Raúl Ortega, se destinơ un pabellón del Servicio de Lactantes, equipado con $8 \mathrm{cu}-$ nas y 1 incubadora, para la atención de prematuros. Este se amplió en 1953, a instancia nuestra, con 2 pequeñas salas para el aislamiento de los infectados, con lo cual su capacidad aumentó a 16 nin̄os. En Septiembre de 1955 se inauguró un Servicio independiente para 25 niños, dotado de todos los elementos necesarios para realizar su atanción integral.

Los datos estadísticos que exponemos en el cuadro N? 1 corresponden al año 1953 , ya completados y rectificados por el Centro de Salud "Independencia". En la imposibilidad de obtener datos exactos de los rubros referentes a prematurez, hemos hecho un cálculo estimativo de ellos, basándonos en investigaciones realizadas con muestras representativas de la Zona. Así, hemos calculado entre los nacidos vivos un $9 \%$ de prematuros y que de éstos fallece el $28,1 \%$ en el curso de primer mes y el $33,3 \%$ en el curso del primer año de su vida (cuadro No 1).
CUADRO TV

DATOS ESTADISTICOS DE LA ZONA NORTE DE SANTIAGO (1953)

(Incluyendo las Circunacripciones de Independencia y Recoleta y las Comanas de Renca, Quilicura, Conchali, Colina, Lampa y Tï-Til)

\begin{tabular}{|c|c|c|}
\hline & & $\begin{array}{c}\text { Tasa por } 1,000 \\
\text { nacidos vivos }\end{array}$ \\
\hline 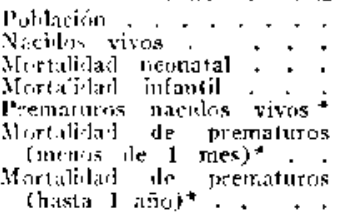 & $\begin{array}{r}291.954 \\
9.9(12 \\
.34 \\
948 \\
882 \\
248 \\
294\end{array}$ & $\begin{array}{l}34 \\
96,7 \\
90 \\
25,3 \\
29,9\end{array}$ \\
\hline
\end{tabular}

Del cuadro anterior puede deducirse que la prematurez determina, directa o indirectamente, el $\mathbf{7 4 , 2} \%$ de la mortalidad neonatal y el $31,0 \%$ de la mortalidad infantil de la Zona. La mortalidad neonatal equivale más o menos a la tercera parte de la mortalidad infantil $y$ de cada 4 niños que fallecen en el primer mes de la vida, 3 son prematuros. Es curioso comprobar que hechos tan llamativos no hayan sido debidamente tomados en cuenta hasta ahora.

Comparativamente, un niño prematuro tiene 30 veces mayores posibilidades de morir en el período neonatal que un niño nacido de término. El insuficiente desarrollo orgánico y la incapacidad relativa de sus funciones vitales básicas, hace necesario que le prodiguemos cuidados especiales para que pueda sobrevivir.

Pero, no todos los niños nacidos prematuros tienen la misma importancia como factor de mortalidad neonatal e infantil. Ello depende fundamentalmente de la duración de la gestación, to que. en forma general, puede apreciarse por el peso de nacimiento. En el cuadro $\mathrm{N}^{\circ}$ 2, hemos reunido 250 casos ingresados al Servicio entre los años 1951 y 1954, los hemos distribuído en los 4 grupos clásicos de Ylppö y hemos estudiado la mortalidad dentro de cada uno de éstos.

CLADRO X? 2

PROPORCION DE FREMATUROS Y MORTALIDAD POR GRUPOS DE PESO

\begin{tabular}{|c|c|c|c|c|c|}
\hline Grupo & Peso do Nac. & N! casos & $\%$ & Fallec. & Mortalidad \\
\hline 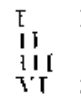 & 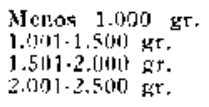 & $\begin{array}{r}9 \\
32 \\
46 \\
163\end{array}$ & $\begin{array}{l}3,6 \\
12,8 \\
18.4 \\
65,2\end{array}$ & $\begin{array}{l}9 \\
17 \\
15 \\
25\end{array}$ & $\begin{array}{l}100 \% \\
59,7 \\
34,7 \\
15,3\end{array}$ \\
\hline & TOTATAS: & 250 & 100,0 & 67 & Prom : $26,8 \%$ \\
\hline
\end{tabular}


Como se vé, a menor peso de nacimiento hay una mayor mortalidad, la que en promedio fué de $26,8 \%$, para los niños hospitalizados en este período. Debemos reconocer que la hospitalización de los prematuros no ha sido todo lo precoz que fuera de desear, ya que sólo un poco más de la cuarta parte de ellos ingresó dentro de las primeras 48 horas de la vida. Si bien es cierto que los internados más tarde son, en cierto modo, los "sobrevivientes" del período más serio por el que debe atravesar un niño prematuro, no lo es menos que los ingresados tarde han llegado, en su mayoria, en malas condiciones generales $o$ infectados, lo que constituye un serio handicap en su contra.

En todo caso estimamos que, con las salvedades expuestas, los datos referidos nos permiten por lo menos tener una idea proporcional de lo que ocurre en la Zona. De ello se deduce que el $31,2 \%$ de los prematuros pesa al nacer entre $1.000 \mathrm{y}$ $2.000 \mathrm{gr} . \mathrm{y}$ es en estos grupos, de mayor mortalidad, donde deben concentrarse los esfuerzos asistenciales, ya que el primer grupo, con reducido número de casos, tiene una mortalidad prácticamente de $100 \%$ y en el cuarto grupo, el más numeroso, la mortalidad es baja y con un buen control domiciliario al principio y de consultorio externo más tarde, basta para que sobrevivan, a menos que padezcan alguna malformación congénita $u$ otra afección que requiera de un tratamiento hospitalario.

\section{RECURSOS ASISTENCIALES}

Estando orientados sobre la magnitud deI problema que vamos a abordar y reconocida su importancia demográfica, veremos a coninuación en qué forma se pueden coordinar los recursos con que contamos en la Zona para darle una solución, lo más completa posible.

No obstante que debamos reconocer que un programa de lucha contra la prematurez debe comenzar por prevenir los partos prematuros, mediante el control médico pre-natal, la educación y la protección de la madre embarazada y una adecuada atención obstétrica del parto ${ }^{1}$, nos limitaremos aquí a abordar el aspecto pediátrico del problema.

Para ello, hemos establecido un plan de trabajo con un objetivo bien definido, partiendo de la base de que, siendo el Hos- pital "Roberto del Rio" el principal centro de asistencia infantil de la Zona, su Servicio de Prematuros debe ser también el rector de la atención pediátrica del niño prematuro, puesto que cuenta con las instalaciones necesarias y el personal entrenado para complir su cometido.

Para poder realizar una labor realmente efectiva, es necesario que actúen coordinadamente los diversos organismos asistenciales que tienen relación con el niño y que son:

1) El Hospital de Nin̈os "Roberto del Río",

2) La Maternidad del Hospital "José J. Aguirre",

3) E1 Centro de Salud "Independencia", $y$

4) Los Consultorios Externos del Servicio Nacional de Salud.

El Hospital "Roberto deI Río" posee una Oficina de Coordinación, a cargo de 2 enfermeras sanitarias, que lo mantiene en estrecha relación con los restantes elementos asistenciales de la Zona.

En el esquema que sigue, se indica la forma en que deberán funcionar coordinadamente estos diversos elementos.

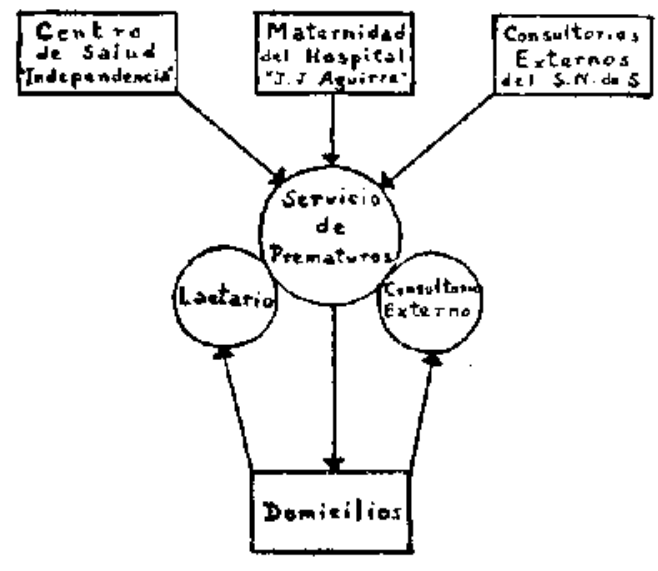

ESQUEMA

Los casos de prematurez serán denunciados al Servicio de Prematuros: a) por el Servicio de Puericultura de la Maternidad del Hospital "José J. Aguirre"; b) por el Centro de Salud "Independencia", que recibirá dentro de las 48 horas que siguen al parto las denuncias formuladas por las matronas domiciliarias controladas y enviará inmediatamente una enfermera sanitaria al hogar; y c) por los Con- 
sultorios Externos del Servicio Nacional de Salud (15 en total). Los pediatras responsables de estos organismos, en los casos a) y c) y las matronas o las enfermeras sanitarias, en el caso b), serán los que discriminarán en cada caso acerca de la conveniencia de hospitalizarlos o de solicitar un control domiciliario al Centro de Salud, el que se hará por medio de enfermeras sanitarias. Como dato ilustrativo podemos agregar que la Maternidad del Hospital "José J. Aguirre" atiende aproximadamente el $40 \%$ de todos los partos de la Zona Norte y que matronas controladas por el Centro de Salud "Independencia" asisten a domicilio más o menos un $10 \%$ de ellos. Por consiguiente, la mitad de los casos de prematurez son susceptibles de una denuncia inmediata. De cumplirse el programa de atención de partos para el próximo año, es de esperar que este porcentaje sea mejorado.

Para desempeñar su cometido, el Servicio de Prematuros del Hospital "Roberto del Río" ha sido planeado con las siguientes secciones: un Consultorio Externo, un Lactario y un Servicio de Hospitalización.

EI Consultorio Externo se halla ubicado en el 1.er piso, tiene una puerta de acceso independiente y consta de una sala de examen y de dos salas de espera, lo que permite separar a los recién nacidos de los niños de mayor edad que vienen a control.

EI Lactario está situado en el subterráneo y se compone de dos piezas separadas por una sala de baño; la primera de éstas se emplea para que las madres se pongan su delantal y mascarilla y la última para que efectúen la extracción de la leche, que se conserva en refrigerador hasta su traslado al servicio de hospitalización.

El Servicio de Hospitalización, ocupa una superficie de más o menos $200 \mathrm{~m} .^{2}$, se encuentra situado en el ala oriente del $5^{\circ}$ piso y está completamente aislado de los restantes Servicios del Hospital. Dividido por un pasillo central, tiene en el lado norte 3 pabellones para hospitalizar los niños y en el lado sur oficina para los médicos y archivo, ropería, cocina de leche y 2 salas de baño. Además cuenta con una pequeña pieza, fuera del Servicio, destinada a la ropa sucia.

Los pabellones de hospitalización, numerados 1,2 y 3 desde la puerta de en- trada, pueden contener en total 25 niños. El pabellón $N^{\circ} 1$ está preparado para atender a los más pequeños, que necesitan calefacción individual. El pabellón $\mathrm{N}^{\circ} 2$ tiene boxes para el aislamiento de Ios infectados. El pabellón No 3 se dedica a los niños que están en buenas condiciones, en espera de alcanzar el peso fijado para su alta. Los niños se intercambian entre ellos de acuerdo con las necesidades del Servicio. Los pabellones disponen de aire acondicionado, central de oxígeno con salidas individuales frente a las incubadoras $\mathrm{y}$ cunas, lavatorios $\mathrm{y}$ baño con agua fría $\mathrm{y}$ caliente y demás mobiliario necesario.

La cocina de leche cuenta con 3 secciones: para la preparación de los alimentos, para el lavado y esterilización de los biberones y para la conservación de la leche humana y de las fórmulas ya preparadas y envasadas.

La ropería tiene estanterias independientes para la ropa que usa el personal del Servicio y para guardar la ropa limpia de los nińos.

El Servicio ocupa el siguiente personal:

1 Médico-jefe.

2 Médicos ayudantes.

1 Enfermera-jefa.

1 Enfermera sanitaria.

12 Auxiliares.

4 Personas de servicio.

1 Mozo.

Los médicos atienden a los niños hospitalizados, el Consultorio Externo y controlan a los hijos de las madres dadoras del Lactario. Concurren al Servicio a diferentes horas del dia y durante la noche los casos de emergencia son atendidos por los médicos resídentes del Hospital.

La enfermera-jefa dirige la labor de enfermería del Servicio. La enfermera sanitaria efectúa el trabajo domiciliario y colabora en el Consultorio Externo.

El personal auxiliar se distribuye en la siguiente forma: 1 sub-jefa del Servicio, 9 para los pabellones, 1 a cargo de la cocina de leche y 1 responsable del Lactario. Las auxiliares de los pabellones hacen turnos rotativos que cubren las 24 horas del día.

El personal de servicio se ocupa de las siguientes labores: 3 para los pabellones y 1 destinada a la ropería y para ayudar en la cocina de leche. El mozo efectúa el aseo del local. 
El trabajo del Servicio se ha standardizado 'siguiendo normas precisas que se han redactado $y$ se entregan por escrito al personal médico, enfermeras, auxiliares y de servicio y cuyo cumplimiento es vigilado estrechamente. Queremos destacar en esta oportunidad la importancia que tíene para la buena marcha de un Servicio de esta índole la especialización del personal, tanto médico como para-médico. Este debe conocer a fondo la técnica de la atención del niño prematuro y contar con un conveniente entrenamiento, para adquirir el cual es necesario darles estabilidad en sus cargos. Solamente con la experiencia que da una larga y continuada práctica, es posible lograr una atención eficierite de estos niños tan delicados, en los que los pequeños detalles son fundamentales.

\section{PLAN DE THABAJO}

El objetivo del Servicio será la atención integral del niño prematuro, con el propósito de disminuír su alta mortalidad e influír, de este modo, en el descenso de los índices de mortalidad neonatal e infantil de la Zona.

Para lograrlo, se ha programado el plan de trabajo que exponemos en seguida.

El Servicio controlará solamente a los niños nacidos con un peso inferior a 2.500 gr. que pertenezcan a la Zona.

Se recibirán para su hospitalización aquellos nińos que, por su poca vitalidad o por presentar complicaciones, requieran de una asistencia especializada y que tengan menos de un mes de edad. Este plazo se ha fijado tomando en consideración que es el período más peligroso para la vida del prematuro y al hecho de que el $50 \%$ de los nacimientos de la Zona escapan a nuestro control inmediato. Serán preferidos los de peso de nacimiento inferior a $2.000 \mathrm{gr}$., los grupos de más alta mortalidad y que tienen mayores posibilidades de sobrevivir con una atención adecuada.

Los niños con peso de nacimiento superior a $2.000 \mathrm{gr}$. serán controlados en el domicilio durante el primer mes de la vida por medio de enfermeras sanitarias, quienes pondrán especial énfasis en los aspectos de calefacción, alimentación $y$ prevención de la infección. Cuando el es- tado general del niño lo permita, seguirán contralados en el Consultorio Externo del Servicio y cuando el caso lo requiera, serán hospitalizados. Si ya han sobrepasado el mes de edad ingresarán al Servicio de Lactantes, en caso de enfermedad.

Se procurará que el ingreso de los niños se haga en lo posible dentro de las primeras 48 horas de la vida, lo que les permite llegar en mejores condiciones y libres de infecciones ${ }^{2}$. Para ello se dará preferencia en la dación de camas a los pedidos de la Maternidad y a las denuncias de las matronas y enfermeras sanitarias del Centro de Salud. Para el traslado se emplearán incubadoras portatiles, de un modelo sencillo, que permiten evitar el enfriamiento del niño. Pero, también se recibirán niños de mayor edad (hasta un mes), porque estimamos que todo prematuro en peligro de morir debe recibir el cuidado adecuado, que sólo puede proporcionarle un Servicio especializado.

Para la atención del niño en el Servicio se seguira una pauta standard "3, sometida sólo a las variaciones individuales que sean estrictamente necesarias. Esto permite hacer un balance de los métodos que se están empleando y corregir los defectos que se hagan evidentes.

Los niños permanecerán hospitalizados hasta que alcancen un peso de $2.500 \mathrm{gr}$. o mientras requieran de algún tratamiento especial médico o quirúrgico. El tiempo de estada podrá prolongarse también, hasta que lleguen a 3.000 gr., cuando las condiciones del hogar sean deficientes, en especial durante los meses fríos del año.

Antes de su alta se solicitará un informe a la enfermera sanitaria del Servicio sobre las condiciones del hogar, a fin de hacer las indicaciones que sean necesarias para la mejor atención del niño en su domicilio, de acuerdo con los medios económicos y la cultura de sus familiares. Una pauta, conteniendo los cuidados básicos a que debe ser sometido y su alimentación, será entregada por escrito.

El control posterior del niño egresado se hará en el Consultorio Externo del Servicio hasta el año de edad, lapso que podrá prolongarse en casos especiales que requieran de una estrecha vigilancia a más largo plazo. La frecuencia de los controles será determinada de acuerdo con 
las necesidades del caso individual, teniendo debida consideración la evolución que siga en el hogar.

Para desarrollar una labor que tenga real influencia sobre la mortalidad del niño prematuro, es necesario cubrir con adecuada atención pediátrica un porcentaje no inferior a $\operatorname{los} 2 / 3$ de los nacidos vivos. Como no podemos pretender hospitalizar a todos los niños prematuros de la Zona, por razones obvias, debemos contentarnos con que ingrese la tercera parte de ellos, los más vulnerables, y realizar un buen control domiciliario y de consultorio externo en otra tercera parte. Estimamos que va a necesitar hospitalización solamente el $60 \%$ de los niños nacidos con peso inferior a 2.000 gr. y el $20 \%$ de los que tengan un peso de nacimiento entre 2.000 y 2.500 gr. Sobre esta base (cuadro $\mathrm{N}^{\circ} 3$ ) llegamos a la cifra de 300 , que serán los prematuros que deberán ingresar anualmente. Contando con capacidad para 25 niños y calculando un promedio de estada de 30 días por cada uno, se puede concluir que pasarán por el Servicio 300 niños por año, lo que resulta suficiente para las necesidades calculadas.

CUADRO NO 3

CALCULO APROXIMADO DE LAS NECESIDADES DEL SERVICIO

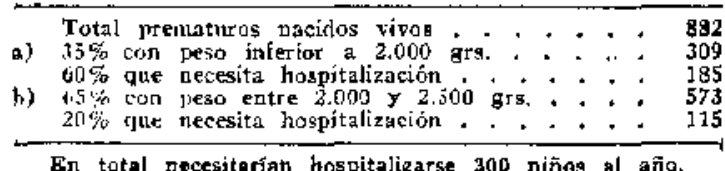

En todo caso, si su capacidad fuera in. suficiente durante los meses de invierno, que son de mayor riesgo para la supervi- vencia de Ios prematuros en el domicilio y durante Ios cuales la estada de los hospitalizados debe prolongarse por las condiciones climatéricas reinantes, podría destinarse, para la atención de niños prematuros de mayor vitalidad, uno o dos pabellones del Servicio de Lactantes, que se halla menos congestionado en esta época del año en que disminuyen enormemente de frecuencia los trastornos nutritivos agudos.

\section{RESUMEN}

Se hace un estudio estadistico del pro. blema de la prematurez en la Zona Norte de Santiago y se propone un plan para la atención del niño prematuro coordinando los diversos organismos asistenciales existentes en ella.

\section{SUMMARY}

THE PROBLEM OF PREMATURE INFANT IN the North Zone of Santiago.

A statistical study on the problem of prematurity in the North Zone of Santiago is made and a plan is proposed for the care of premature infants by means of a coordination of the different organizations of medical asistance existing in $t i$.

\section{BIBLIOGRAFÍA}

1.-AVENLANO, 0 - "Tratauiento pretentipo $y$ con. ducta obstétrica en el parto irematuro". Rev. Chilena de Ped. $25 \div 482,1954$

2. GARCES, H.; MUAOZ, R. F FERNANDER, F.-"I. "nfección en el niño prematriro". Kev. Chilenis de Pecl. $25=109,1954$.

3.-GARCES. H. - "Esquema ile tratamietito del niño prunatero" Rev. Chilena de Ped. 26:479, 1955 .

4.- WALLGRF, A, - "Estudios store los yrematuros", fil Keciell Nacido, $2: 140,1954$. 